\title{
Repräsentations- und Entscheidungskulturen im Vergleich: Die Parteitage von FDP und Grünen
}

\author{
Deniz Anan
}

Die Parteien in Deutschland scheinen einander in organisatorischer Hinsicht stark angenähert. Grundgesetz, Parteiengesetz, die einschlägige Rechtsprechung des Bundesverfassungsgerichts und die Erfordernisse der modernen (Medien-)Demokratie üben eine stark vereinheitlichende Wirkung aus. ${ }^{1}$ Hinzu kommt der spezifische Einfluss von Internet und sozialen Netzwerken. ${ }^{2}$ Dies gilt vor allem, seitdem sich die Grünen, deren basisdemokratisches Alternativkonzept ursprünglich stark kontrastierte, den übrigen Parteien in mehreren Parteistrukturreformen angenähert haben. Dennoch sind, so die hier vertretene Ausgangsthese, bei genauerem Hinsehen signifikante organisatorisch-kulturelle Unterschiede auszumachen, die insbesondere auf die unterschiedliche Herkunft und das Selbstverständnis einer Partei zurückzuführen sind.

Organisatorische und strukturelle Fragen haben früh das Interesse der Parteienforschung geweckt. Die Klassiker Robert Michels und Moissei Ostrogorski wie auch Maurice Duverger rückten das Moment einer oligarchischen Tendenz innerparteilicher Demokratie in den Fokus. ${ }^{3}$ Zeitgenössische Autoren diskutieren, ob sich die Parteien von Volksparteien zu professionellen Wähler- beziehungsweise Kartellparteien wandeln oder der Typ der Mitgliederpartei nach wie vor relevant ist. ${ }^{4}$ Parteityp, Organisation und Selbstverständnis stehen also in einem engen Zusammenhang zueinander.

In der Organisationstheorie wird Organisationskultur als ein erlerntes, bewährtes und bindendes Muster gemeinsamer Grundprämissen (Werte, Normen, Annahmen) verstanden, das Organisationen bei der Lösung innerer (Integration) und äußerer Probleme (Adaption)

1 Vgl. Thomas Poguntke, Parteiorganisation im Wandel, Wiesbaden 2000; Marion Müller, Parteitage in der Mediendemokratie, in: Ulrich von Alemann / Stefan Marschall, Parteien in der Mediendemokratie, Wiesbaden 2002, S. 147 - 172; Ulrich Sarcinelli, Parteien in der Kommunikationsfalle? Zwischen politischem Traditionsverein und Event-Agentur, in: ders. / Jens Tenscher (Hrsg.), Machtdarstellung und Darstellungsmacht, Beiträge zu Theorie und Praxis moderner Politikvermittlung, Baden-Baden 2003, S. 49 - 60; Uwe Jun, Der Wandel von Parteien in der Mediendemokratie, Frankfurt am Main 2004.

2 Vgl. Christoph Bieber (Hrsg.), Parteipolitik 2.0 - Der Einfluss des Internet auf parteiinterne Kommunikations- und Organisationsprozesse, Bonn 2001; Stefan Marschall, Parteien und Internet Auf dem Weg zu internet-basierten Mitgliederparteien, in: APuZ, 51. Jg. (2001), H. 10, S. 38 46.

3 Vgl. Robert Michels, Zur Soziologie des Parteiwesens in der modernen Demokratie, Stuttgart 1989; Moissei Ostrogorski, La Démocracie et les Partis Politiques, Paris 1993; Maurice Duverger, Les Partis Politiques, Paris 1951.

4 Vgl. Peter Haungs, Plädoyer für eine erneuerte Mitgliederpartei, in: ZParl, 25. Jg. (1994), H. 1, S. 108 - 115; Richard Katz / Peter Meir, Changing Models of Party Organization and Party Democracy, in: Party Politics, 1. Jg (1995), H. 1, S. 5 - 28; Klaus von Beyme, Funktionswandel der Parteien in der Entwicklung von der Massenmitgliederpartei zur Partei der Berufspolitiker, in: Oscar W. Gabriel / Oskar Niedermayer / Richard Stöss (Hrsg.), Parteiendemokratie in Deutschland, Bonn 1997, S. 359 - 382; Elmar Wiesendahl, Parteien in Perspektive, Opladen 1998; Klaus von Beyme, Parteien im Wandel, Wiesbaden 2000. 
hilft. Organisationskultur ist auf drei Ebenen fassbar: Unter der sichtbaren oberen Ebene der so genannten Artefakte, zu denen auch Verhaltensweisen und Erzeugnisse jeder Art (zum Beispiel Programme, Logos) zählen, liegen die öffentlich propagierten Werte (mittlere Ebene) und die unausgesprochenen gemeinsamen Grundannahmen (unterste Ebene). ${ }^{5}$ Kulturellen Faktoren wird eine große Bedeutung für organisatorische Stabilität und Wandel zugeschrieben. ${ }^{6}$ An dieses Verständnis wird im Folgenden angeknüpft: Auch unter der sichtbaren Oberfläche eines Parteitags und der ihn steuernden Regeln (Satzung, Geschäftsordnung) stehen Werte und gewohnheitsmäßig angewandte, geteilte und kaum hinterfragte Muster. Somit erscheint es sinnvoll, die Repräsentations- und Entscheidungsfindungsaspekte von Parteitagen, die im Mittelpunkt der Analyse stehen, als Repräsentations- beziehungsweise Entscheidungskulturen zu begreifen.

Verglichen werden die einschlägigen Regelungen bei FDP und Bündnis 90/Die Grünen, und zwar jeweils im Bundesverband und, exemplarisch, im Landesverband Bayern. ${ }^{7}$ Diese Auswahl erfolgte, weil hier, so die Vermutung, ein klassisch liberales Repräsentationsmodell einem - zumindest ursprünglich einmal - alternativen basisdemokratischen Repräsentationsmodell gegenübersteht. Die Untersuchung reiht sich außerdem in politikwissenschaftliche Vergleiche dieser beiden Parteien ein $^{8}$, was angesichts des augenfälligen Nebeneinanders großer Gemeinsamkeiten (ähnliche Wählerschichten, Bestandteile der neuen Koalitionsmodelle Ampel- und Jamaikakoalition) und deutlicher Unterschiede (Einstellungen der Wählerschaft, gegenseitige Betrachtung als Hauptgegner) nahe liegt.

Die Hypothese lautet, dass die unterschiedlichen Repräsentions- und Entscheidungskulturen der Parteitage die unterschiedliche Herkunft der Parteien jeweils klar widerspiegeln: Bei der FDP darf ein klassisches liberales Repräsentations- und Entscheidungsmodell und ein Nachwirken der Herkunft als Honoratiorenpartei vermutet werden. Bei den Grünen hingegen werden trotz aller Strukturreformen ein signifikanter fortbestehender Einfluss des alternativen Konzepts der Basisdemokratie und der Herkunft als Anti-Parteien-Partei vor dem Hintergrund der Neuen Sozialen Bewegungen erwartet. ${ }^{9}$ Im Vordergrund stehen folglich die mittlere und untere Ebene der Organisationskultur, denn hier dürften sich Selbstverständnis und Entstehungshintergrund am ehesten noch auswirken.

Methodisch soll diese Frage durch eine vergleichende Analyse der Satzungsgrundlagen, also der Bundes-, Landes- und ausgewählter Kreissatzungen beantwortet werden, unter Einbezug von Annexen (Geschäftsordnungen, Wahlordnungen usw.) und Erläuterungen in der Online-Selbstdarstellung der Parteien. ${ }^{10}$ Experteninterviews mit Mitarbeitern der Partei-

5 Vgl. Edgar Schein, Organisationskultur, Bergisch Gladbach 1999.

6 Vgl. Judi Marshall / Adrian McLean, Exploring Organisation Culture as a Route to Organisational Change, in: Valerie Hammond (Hrsg.), Current Research in Management, London 1985, S. 3 - 20.

7 Wenn nichts anderes angegeben, ist in der Folge immer der Landesverband Bayern gemeint.

8 Vgl. Franz Walter, Gelb oder Grün? Kleine Parteiengeschichte der besserverdienenden Mitte in Deutschland, Bielefeld 2010.

9 Vgl. Dietrich Herzog, Die Führungsgremien der Parteien: Funktionswandel und Strukturentwicklungen, in: Oscar W. Gabriel / Oskar Niedermayer / Richard Stöss (Hrsg.), a.a.O. (Fn. 4), S. 301 - 322; Thomas Poguntke, a.a.O. (Fn. 1).

10 Es handelt sich um die Satzungen und Geschäftsordnungen des FDP-Bundesverbands und des FDP-Landesverbands Bayern sowie des Grünen-Bundesverbands, der Grünen-Landesverbände Bayern, Baden-Württemberg, Berlin, Hamburg und Hessen sowie der Grünen-Stadtverbände München und Augsburg. 
geschäftsstellen ${ }^{11}$ und die teilnehmende Beobachtung von Parteitagen ${ }^{12}$ ergänzen die Analyse.

\section{Parteitage: Typologie und Funktionen}

Hinter der einheitlichen Bezeichnung „Parteitag“ verbergen sich unterschiedliche Versammlungsformen. So lassen sich ordentliche und außerordentliche Parteitage unterscheiden, außerdem die Versammlungen zur Aufstellung von Listen im Vorfeld von öffentlichen Wahlen.

Parteitage sind laut Parteiengesetz die obersten Organe der Parteien. Sie verfügen über die höchste Legitimation. Vorstände und weitere Gremien leiten ihre Legitimation von der Wahl oder die Bestätigung durch den Parteitag ab. Üblicherweise ${ }^{13}$ werden folgende weitere Funktionen unterscheiden: (1) personelle Entscheidung, (2) sachliche Entscheidung, (3) programmatische Entscheidung beziehungsweise Planung, (4) Wahlwerbung und (5) Repräsentation. ${ }^{14}$ Hier werden alle Funktionen außer der Wahlwerbung berücksichtigt, wobei die ersten drei Funktionen für die Entscheidungskultur und die fünfte für die Repräsentationskultur von Bedeutung sind. Durch die enge Durchdringung von Parteien und Staat beeinflussen Parteitage auch die parlamentarisch-gouvernementale Willensbildung. ${ }^{15}$

Die verschiedenen Funktionen lassen sich unterschiedlichen Parteitagstypen zuordnen: Außerordentliche Parteitage dienen der programmatischen Diskussion einer spezifischen Frage (Programmfunktion), der Billigung des Koalitionsvertrags (Sachentscheidungsfunktion) oder der programmatischen und koalitionspolitischen Festlegung im Vorfeld von Wahlen (Wahlkampffunktion). In letzterem Fall steht die mediale Inszenierung im Vordergrund; Parteitage nähern sich den aus den USA bekannten „Krönungsparteitagen "16 beziehungsweise „Krönungsmessen “17 an. Ordentliche Parteitage lassen sich in Wahlparteitage und

11 Interview mit Martin Hagen, Hauptgeschäftsführer FDP Bayern und Emmerich Frößl, Leiter Parteienrecht und Verwaltung, Landesgeschäftsstelle FDP Bayern, 13. Februar 2011, ergänzt durch Nachträge; Interview mit Fabian Hamák, Landesgeschäftsführer Grüne Bayern, 14. Februar 2011, ergänzt durch Nachträge; Schriftliche Anfrage bei Dorothea Staiger, Grünen-Bundesgeschäftsstelle.

12 FDP: LPT 2000 in Lauingen, BPT 2000 in Nürnberg, LPT 2000 in Dingolfing, BPT 2001 in Düsseldorf, LPT 2002 in Bad Kissingen, BPT 2003 in Bremen, LPT 2004 in Lindau, LPT 2006 in Würzburg, LPT 2007 in Memmingen; Grüne: LPT 2008 in Rosenheim, BPT 2008 in Erfurt, BPT 2009 in Dortmund, LPT 2009 in Bamberg, LPT 2010 in Würzburg, LPT 2011 in Bad Windsheim.

13 Vgl. Heino Kaack, Geschichte und Struktur des deutschen Parteiensystems, Opladen 1971.

14 Vgl. Hans Schuster, Die Heerschau der Parteien, Theorie und Praxis der Parteitage, in: Politische Studien, 8. Jg. (1957), H. 88/89, S. 57 - 92; Jürgen Dittberner, Funktionen westdeutscher Parteitage, in: Otto Stammer (Hrsg.), Parteiensysteme, Parteiorganisationen und die neuen politischen Bewegungen, Berlin 1968, S. 116 - 128; Jürgen Dittberner, Die Bundesparteitage der CDU und der SPD von 1946 bis 1968 - Eine Untersuchung der Funktionen von Parteitagen, Augsburg 1969; Wolfgang Steiner, SPD-Parteitage 1964 und 1966 - Analyse und Vergleich, Meisenheim 1970; Joachim Raschke, Parteitage der Grünen, in: APuZ, 41. Jg. (1991), H. 11/12, S. 46 - 54.

15 Vgl. Dietrich Herzog, a.a.O. (Fn. 9).

16 Christina Holtz-Bacha, Wahlkämpfe in Deutschland, in: Andreas Dörner / Ludgera Vogt, WahlKämpfe, Frankfurt am Main 2002, S. 211 - 231.

17 Hans-Georg Soeffner / Dirk Tänzler, Medienwahlkämpfe - Hochzeiten ritueller Politikinszenierung, in: Andreas Dörner / Ludgera Vogt, a.a.O. (Fn. 16), S. $92-115$. 
Arbeitsparteitage unterteilen, je nachdem ob die Neuwahl des Vorstands (Personalentscheidungsfunktion) oder die inhaltliche Diskussion (Sachentscheidungs- beziehungsweise Programmfunktion) im Vordergrund steht. ${ }^{18}$ Die Freiheit der Delegierten zur autonomen Entscheidung und zur Kontrolle sollte nicht überschätzt werden ${ }^{19}$, wie Heino Kaacks Zöllner-Analogie wohl am anschaulichsten auf den Punkt bringt: Danach inspizieren Parteitage insbesondere bei Sach- und Programmentscheidungen wie Zöllner die Inhalte von Reisekoffern, können hierüber aber nicht frei verfügen. Dies gilt trotz oder vielleicht gerade wegen des basisdemokratischen Anspruchs auch für die Parteitage der Grünen. ${ }^{20}$

\section{Repräsentations- und Entscheidungskulturen auf Parteitagen im Vergleich}

\subsection{Das basisdemokratische Alternativkonzept der Grünen ${ }^{21}$}

In ihrem ersten Grundsatzprogramm bekannten sich die Grünen 1980 zu dem Ziel, „eine Parteiorganisation neuen Typs“ zu schaffen. Dieses basisdemokratische Alternativkonzept enthielt das Rotationsprinzip, wonach Abgeordnete nach einer festgelegten Zeitspanne (zumeist zwei Jahre) ihr Mandat zurückgeben sollten. Machtbegrenzend sollten außerdem die Mehrfachspitze von bis zu drei Vorsitzenden und die Trennung von (Partei-)Amt und (Abgeordneten-)Mandat wirken. Zur Frauenquote (ungerade Listenplätze bei öffentlichen und parteiinternen Wahlen sind Frauen vorbehalten) trat 1993 infolge der Fusion mit Bündnis 90 eine überproportionale Ostdeutschenquote (vier Neuntel) im Bundesvorstand. Das Repräsentations- und Entscheidungsverständnis kam außerdem in der Verwendung alternativer Bezeichnungen wie „Sprecher“ anstelle von „Vorsitzendem“ zum Ausdruck. Repräsentation bei den Grünen näherte sich dem imperativen Mandat an. Parteitage fanden häufiger als üblich statt, auf Landesebene teils als Mitgliederversammlungen; Vertreter von Umwelt- und Bürgerinitiativen hatten auf Parteiversammlungen jederzeit Rede- und Antragsrecht.

Das Basisdemokratie-Konzept der Grünen bezieht sich nicht nur auf die obere Organisationsebene des sichtbaren Verhaltens (zum Beispiel Abgabe eines Basisvotums), sondern lässt sich auch auf der mitteleren Ebene der propagierten Werte (zum Beispiel Basisorien-

18 Vgl. Marion Müller, Parteitagskommunikation: Funktionen, Strukturen, Trends von Parteitagen in Deutschland und in den USA, in: Heribert Schatz / Patrick Rössler / Jörg-Uwe Nieland (Hrsg.), Politische Akteure in der Mediendemokratie, Wiesbaden 2002, S. 65 - 77.

19 Vgl. Ulrich Lohmar, Innerparteiliche Demokratie, Stuttgart 1963; Wolfgang Abendroth, Das Problem der innerparteilichen und innerverbandlichen Demokratie in der Bundesrepublik, in: ders. (Hrsg.), Antagonistische Gesellschaft und politische Demokratie. Aufsätze zur politischen Soziologie, Neuwied / Berlin 1967, S. 272 - 317.

20 Vgl. Joachim Raschke, a.a.O. (Fn. 14); Dieter Salomon, Grüne Theorie und graue Wirklichkeit Die Grünen und die Basisdemokratie, Freiburg 1992; Sebastian Bukow / Stephan Rammelt, Parteimanagement vor neuen Herausforderungen, Münster 2003; Christin Leistner / Katharina Rahlf, Grün bleibt die Hoffnung? Die Bündnisgrünen zwischen Harmonie und Krise, in: Felix Butzlaff I Stine Harm / Franz Walter (Hrsg.), Patt oder Gezeitenwechsel? Deutschland 2009, Wiesbaden 2009, S. $129-155$.

21 Vgl. Ralf Heidger, Die Grünen. Basisdemokratie und Parteiorganisation, Berlin 1987; Dieter Salomon, a.a.O. (Fn. 20). 
tierung, Bindung der Repräsentanten an den Mitgliederwillen) und der unteren Ebene der selten ausgesprochenen Grundannahmen (zum Beispiel die Überzeugung, dass es gerecht ist, Listen überwiegend mit Frauen zu besetzen, oder Macht nach zwei Jahren abzugeben) verorten.

Nach mehreren Parteistrukturreformen ergibt sich heute folgendes Bild: Die Grünen kennen kein formales Rotationsprinzip mehr; informelle Reste bestehen hingegen fort, zum Beispiel wenn die langjährige Zugehörigkeit zu Parlamenten bei der Listenaufstellung aus Delegiertensicht einen Malus darstellt. Die Doppelspitze gibt es nach wie vor auf allen Ebenen. Bezeichnungen wie „Sprecher“ haben sich auf den unteren Ebenen teils halten können, wurden größenteils aber durch die herkömmliche Terminologie ersetzt. Die Trennung von Amt und Mandat wurde unterschiedlich stark gelockert, aber meist beibehalten: In der Regel darf ein Teil des Vorstands über ein Abgeordnetenmandat verfügen. Die Frauenquote hat ungeachtet regelmäßiger Kritik seitens männlicher Grüner in unveränderter Form Bestand. Ostdeutsche sind seit 1998 in den Bundesgremien nur noch gemäß ihrem Anteil an der Bevölkerung nach vertreten. Umweltinitiativen haben kein automatisches Rederecht mehr, aber für zwölf Monate ist eine „freie Mitarbeit“ bei den Grünen möglich, ohne Parteimitglied zu sein. Das basisdemokratische Alternativkonzept wurde also teils aufgegeben, teils modifiziert und teils beibehalten.

\subsection{Rahmenbedingungen der Parteitage}

Die FDP verwendet durchgehend die Bezeichnung „Parteitag“, die Grünen alternative Begriffe wie „Versammlung“ oder „Delegiertenkonferenz“. Turnus (meist einmal jährlich) und Tagungsdauer (je nach Ebene ein bis drei Tage am Wochenende) sind bei beiden Parteien ähnlich. Die Parteitage stehen jeweils allen Mitgliedern offen, bei den Grünen teils auch Vorstandssitzungen. Der Tagungsort rotiert zumeist. Kriterien sind die gleichmäßige Berücksichtigung aller Landesteile und die Herstellung von Medienöffentlichkeit vor Landtagswahlen. ${ }^{22}$ Erreichbarkeit und Bezahlbarkeit der Tagungshalle sind ab der Landesebene weitere wichtige Kriterien.

Das Umfeld der Parteitage ähnelt sich ebenfalls: Ab der Landesebene sind Parteitage durch eine professionelle Regie und begleitende Medienarbeit geprägt. Das Personal der Tagungspräsidien besteht aus Mitgliedern, die einerseits über Erfahrung und Professionalität verfügen, andererseits aber kein hohes Parteiamt haben, und ist daher von großer Kontinuität geprägt. In der Vorhalle, der sprichwörtlichen Lobby, präsentieren sich Parteigruppierungen und externe Aussteller, bei den Grünen überwiegend mit ökologischem Hintergrund.

22 Dieses Muster ist nicht durchgehend sicht-, aber erkennbar: So fanden FDP-Bundesparteitage zuletzt 2003 (Bremen), 2004 (Dresden), 2005 (Köln), 2006 (Rostock), 2008 (München), 2010 (Köln) und 2011 (Rostock) in Ländern mit bevorstehenden Landtagswahlen statt; Grünen-Bundesdelegiertenkonferenzen analog 2002 (Hannover), 2003 (Dresden), 2007 (Göttingen/Nürnberg), 2008 (Erfurt), 2010 (Freiburg), 2011 (Kiel) und 2012 (Hannover). 


\subsection{Delegiertenwahlverfahren}

Die Delegiertenwahl kann trotz Normierung durch Parteiengesetz und Rechtsprechung unterschiedlich ausgestaltet werden. So werden auf Kreisebene bei den Grünen die Landesund Bundesparteitagsdelegierten gewählt, bei der FDP nur die Landesparteitagsdelegierten. Die Bundesparteitagsdelegierten werden bei den Liberalen in einem doppelten Wahlverfahren nominiert: faktisch auf Bezirks-, nominell aber auf Landesebene. ${ }^{23}$ Die Implikationen sind weitreichend: Bei der FDP ist die persönliche Bekanntheit auf Bezirksebene $^{24}$ ein entscheidendes Wahlkriterium, bei den Grünen ist es hingegen die Verwurzelung vor Ort.

Auch die Zuteilung der Delegiertenmandate erfolgt unterschiedlich: Die FDP teilt die Delegiertenzahlen jeweils zur Hälfte nach dem Mitgliederanteil und nach dem Wählerstimmenanteil auf ${ }^{25}$; ein Grundmandat jedes Kreisverbands gibt es nur auf Bezirks- und Landesebene. Die Grünen berechnen die Delegiertenzahlen nur nach dem Mitgliederanteil. Auf dem Landesparteitag gibt es zwei, auf dem Bundesparteitag ein Grundmandat für jeden Kreisverband. Ein FDP-Bundesparteitag besteht aus 662, ein Landesparteitag aus 420 bis 427, ein Bezirksparteitag aus 28 (Oberpfalz) bis 166 (Oberbayern) Delegierten. Die Grünen-Bundesdelegiertenkonferenz 2011 umfasste 819 Mandate, die Landesversammlung 340, die Bezirksversammlungen 27 bis 116. Die FDP kennt feste Delegiertenzahlen. Bei den Grünen ist im Bund die tatsächliche Delegiertenzahl infolge der Grundmandate höher als die Richtzahl 750. Auf Landes- und Bezirksebene werden den Kreisverbänden in einem degressiv-proportionalen Verfahren über die zwei Grundmandate hinaus je nach Mitgliederzahl weitere Delegierte zugewiesen. Das Zuteilungsverfahren wurde 2011 vor dem Hintergrund starken Mitgliederwachstums und der in Bayern mit 89 besonders hohen Zahl der Kreisverbände mit dem Ziel sinkender Gesamtgröße modifiziert.

Aufgrund der Grundmandate der Kreisverbände lassen sich Grünen-Parteitage eindeutig dem Basisdemokratie-Modell zuordnen, wobei sich angesichts der Relativierung des Demokratieprinzips (Gleichheit jeder Stimme beziehungsweise jedes Mitglieds) durch die Vertretung aller Gliederungen unabhängig von deren Größe allerdings die normative Frage nach dem demokratischen Charakter stellen lässt. FDP-Parteitage folgen dem Modell individueller, repräsentativdemokratischer Repräsentation. Hinsichtlich des Berechnungsverfahrens steht der Mitglieder- und Wählerorientierung (FDP) die reine Mitgliederorientierung (Grüne) gegenüber. Die Freidemokraten scheinen also, vorsichtig formuliert, dem Modell der Wählerpartei näher zu stehen als die Grünen.

23 Die genaue Formulierung lautet: „Im Landesparteitag sind bei den Delegiertenwahlen zum Bundesparteitag (...) die Bezirksparteitage in dem Verhältnis vorschlagsberechtigt, in dem Delegierte aus ihrem Bereich, beim Landesparteitag stimmberechtigt sind. Weitere Vorschläge können aus der Mitte des Landesparteitags kommen." (FDP Bayern, Satzung, Wahlordnung, § 3); vgl. Heino Kaack, a.a.O. (Fn. 13), S. 521.

24 Der größte bayerische Bezirk, Oberbayern, umfasst immerhin rund $17.500 \mathrm{~km}^{2}$ und über vier Millionen Einwohner, ist also hinsichtlich Einwohnerzahl beziehungsweise Fläche größer als neun beziehungsweise sechs eigenständige Bundesländer.

25 Grundlage ist das Bundestagswahlergebnis (Bundesparteitag) beziehungsweise das Landtagswahlergebnis (Bezirks-/Landesparteitag). 


\subsection{Statistische Daten ${ }^{26}$}

(1) FDP und Grüne sind in quantitativer Hinsicht (Mitgliederzahl, -anteile, Delegationsgröße) und in der Verteilung über die Bezirke, Städte und Landkreise sehr ähnlich. München-Stadt und das Münchener Umland sind Mitgliederschwerpunkte beider Parteien. Hochburgen sind der Landkreis Starnberg mit der bundesweit höchsten Millionärsdichte ${ }^{27}$ (FDP) beziehungsweise der Landkreis Fürstenfeldbruck (Grüne). Diaspora ist jeweils die Peripherie Nord- und Ostbayerns.

(2) Beide Parteien sind im Bund überproportional stark vertreten - die FDP wegen des überdurchschnittlichen Bundestagswahlergebnisses ${ }^{28}$, die Grünen aufgrund der hohen Zahl an Kreisverbänden und des Grundmandats.

(3) Wegen des Grundmandates sind bei den Grünen auch im kleinsten Bezirk Oberpfalz alle Kreisverbände auf dem Bundesparteitag vertreten, bei der FDP hingegen nur jeder zweite Oberpfälzer Kreisverband. Bei den Grünen weichen Mitglieder- und Delegiertenanteile daher stärker voneinander ab.

(4) Setzt man Mitglieder- und Delegiertenzahl zueinander ins Verhältnis, um den genauen Grad der Über- beziehungsweise Unterrepräsentation messen zu können, erhält man im Falle der FDP eine Bandbreite von 10,0 Mitgliedern pro (Landesparteitags-)Delegiertem im Kreisverband Lichtenfels bis 19,2 Mitgliedern pro Delegiertem im Kreisverband MünchenStadt. Der Abweichungsfaktor beträgt 1,9. Die entsprechende Abweichung bei den Grünen liegt viel höher (Faktor 3,45), weil die Kreisverbände hier zwei Grundmandate haben und die degressiv-proportionale Verteilung die mitgliederstarken Kreisverbände relativ benachteiligt. Auf Bezirksebene ergeben sich ähnliche Werte: In der FDP entfallen 11,1 bis 16,0 Mitglieder auf einen Landesparteitagsdelegierten (Abweichungsfaktor 1,44) und 62,4 bis 82,8 Mitglieder auf einen Bundesparteitagsdelegierten (Abweichungsfaktor 1,32); bei den Grünen sind es 21,5 bis 29,1 Mitglieder pro Landesparteitagsdelegiertem (Abweichungsfaktor 1,34) und 41,5 bis 66,3 Mitglieder pro Bundesparteitagsdelegiertem (Abweichungsfaktor 1,6). Die Abweichung ergibt sich durch Rundung und Grundmandate. ${ }^{29}$ Das Grundmandat erklärt auch die stärkere Abweichung bei Grünen-Bundesparteitagsdelegationen auf Bezirks- und insbesondere auf Kreisebene (18,0 bis 68,9 Mitglieder pro Delegiertem; Abweichungsfaktor 3,83). Eine Bundesdelegiertenkonferenz der Grünen ist also in quantitativdistributiver Hinsicht ein ganz anderes Gremium als ein FDP-Bundesparteitag.

\subsection{Delegiertenamt}

Die FDP wählt Delegierte für zwei Jahre, die Grünen für eine konkrete Versammlung. FDPDelegierte können ihre Stimme übertragen und bis zu zwei Stimmen gleichzeitig ausüben.

26 Stichtag ist der 31. Dezember 2010 (Angaben Bundesverband Grüne) beziehungsweise der 31. März 2011 (Angaben FDP) beziehungsweise der 30. Juni 2011 (Angaben Landesverband Grüne).

27 Vgl. Stephan Lebert, Die Starnberger Republik, in: Die Zeit vom 20. Dezember 2006.

28 Vgl. Der Bundeswahlleiter, Endgültiges Ergebnis der Bundestagswahl 2009, http://www.bundeswahlleiter.de/de/bundestagswahlen/BTW_BUND_09/ergebnisse/bundesergebnisse/ (Abruf am 28. Juni 2012).

29 Bei den Grünen haben die beiden unterschiedlichen Stichtage einen zusätzlichen geringen Verzerrungseffekt. 


\begin{tabular}{|c|c|c|}
\hline & FDP & Grüne \\
\hline $\begin{array}{l}\text { Mitgliederzahl/-anteil Landesverband (LV) } \\
\text { Bayern }\end{array}$ & $6.200(9,8 \%)$ & $8.020(13,6 \%)$ \\
\hline Mitglieder Bezirksverband (BzV) Oberbayern & $2.650(42,8 \%)$ & $3.381(42,2 \%)$ \\
\hline Mitglieder BzV Oberpfalz & $312(5,0 \%)$ & $580(7,2 \%)$ \\
\hline Mitglieder Stadtverband (SV) München & $1.112(18,0 \%)$ & $1.207(15,0 \%)$ \\
\hline Mitglieder KV München-Land & $313(5,2 \%)$ & $331(4,4 \%)$ \\
\hline Mitglieder KV Starnberg & $222(3,6 \%)$ & $172(2,1 \%)$ \\
\hline Mitglieder KV Fürstenfeldbruck & $105(1,7 \%)$ & $223(2,8 \%)$ \\
\hline Mitglieder KV Amberg & & $20(0,2 \%)$ \\
\hline Mitglieder KV Lichtenfels & $10(0,2 \%)$ & \\
\hline Gesamtzahl Bundesparteitagsdelegierte & 662 & 819 \\
\hline Bundesparteitagsdelegierte LV Bayern & $82(12,4 \%)$ & $125(15,3 \%)$ \\
\hline davon Delegierte BzV Oberbayern & $32(39,0 \%)$ & $46(36,8 \%)$ \\
\hline davon Delegierte BzV Oberpfalz & $5(6,1 \%)$ & $12(9,6 \%)$ \\
\hline Kreisverbände BzV Oberbayern (Anzahl) & 23 & 22 \\
\hline Kreisverbände BzV Oberpfalz (Anzahl) & 10 & 9 \\
\hline \multicolumn{3}{|l|}{ Mitglieder / Bundesparteitagsdelegierter } \\
\hline KV Amberg-Stadt & Berechnung nicht möglich & 18,0 \\
\hline SV München & Berechnung nicht möglich & 68,9 \\
\hline Abweichungsfaktor kleinster/größter KV & Berechnung nicht möglich & 3,83 \\
\hline BzV Oberpfalz & 62,4 & 41,5 \\
\hline BzV Oberbayern & 82,8 & 66,4 \\
\hline Abweichungsfaktor kleinster/größter BzV & 1,32 & 1,6 \\
\hline Gesamtzahl Delegierte Landesparteitag & 421 & 308 \\
\hline davon Delegierte BzV Oberbayern & $166(39,4 \%)$ & $116(37,8 \%)$ \\
\hline davon Delegierte BzV Oberpfalz & $28(6,7 \%)$ & $27(8,8 \%)$ \\
\hline \multicolumn{3}{|l|}{ Mitglieder / Landesparteitagsdelegierter } \\
\hline KV Lichtenfels & 10,0 & - \\
\hline KV Amberg-Stadt & - & 10,0 \\
\hline SV München & 19,2 & 34,5 \\
\hline Abweichungsfaktor kleinster/größter KV & 1,9 & 3,45 \\
\hline BzV Oberpfalz & 11,1 & 21,5 \\
\hline BzV Oberbayern & 16,0 & 29,1 \\
\hline Abweichungsfaktor kleinster/größter BzV & 1,44 & 1,34 \\
\hline
\end{tabular}

Die Grünen kennen weder Stimmrechtsübertragung noch Mehrfachstimmrecht. Bei ihnen werden Fahrt- und Übernachtungskosten voll erstattet und den Delegierten direkt ausbezahlt. FDP-Delegierte verzichten hingegen auf die Auszahlung. Die Kosten gelten als steuerlich begünstigte Parteispende und werden den einkommensteuerpflichtigen Delegierten teilweise und indirekt erstattet. Während es bei den Grünen vorkommen kann, dass Delegierte bei der Wahl oder im Nachhinein nach ihrem Abstimmungs- oder Wahlverhalten gefragt werden, gibt es eine derartige Ex-Post- beziehungsweise Ex-Ante-Verantwortlichkeit bei der FDP nicht. 
Ein Delegierter bekleidet bei der FDP also ein persönliches Amt: Er ist auf Zeit gewählter, individueller Repräsentant. Bei den Grünen hingegen ist das Delegiertenamt viel stärker funktional: Der Delegierte ist Mandatar der Parteibasis.

Der Frauenanteil an den Mitgliedern der FDP ist stark unterdurchschnittlich (23 Prozent). ${ }^{30}$ Auch kennen die Liberalen keine Frauenquote. Bei den Grünen stehen ungerade Plätze hingegen immer Frauen zu. Diese Regelung müsste sich aufgrund der niedrigen Gesamtzahl der Delegierten in kleinen Kreisverbänden theoretisch erheblich auswirken. Allerdings gilt die Frauenquote nicht, wenn nur ein einziger Platz zur Verfügung steht. Auch werden Frauenplätze meist für Männer „geöffnet“, wenn sich nicht genügend Kandidatinnen finden. Bei Landesparteitagen beträgt der Frauenanteil etwa 40, bei Bundesparteitagen 46 bis 49 Prozent. Der Frauenanteil steigt mit der Delegationsgröße: Kreisverbände mit nur einem Delegiertenmandat entsenden zu 38,5 Prozent Frauen, Kreisverbände mit zwei oder mehr Mandaten zu 53 Prozent.

Sowohl die FDP als auch die Grünen ergänzen die in Wahlen vergebenen Delegiertenmandate durch Ex-Officio-Mandate: Auf Landesparteitagen sind die Landesvorstandsmitglieder und die Bezirksvorsitzenden qua Amt Delegierte. Auf FDP-Bundesparteitagen gibt es umfangreiches Ex-Officio-Rederecht, unter anderem für den Bundesvorstand, für Bundestagsabgeordnete und Vorsitzende der Sonderorganisationen. Sonst dürfen nur gewählte Delegierte reden. Auf Grünen-Bundesdelegiertenkonferenzen haben alle Mitglieder Rederecht; es gibt keine Ex-Officio-Delegierte.

\subsection{Antrags- und Einberufungsrecht}

In beiden Parteien existieren umfangreiche Antrags- und Einberufungsrechte. Bei der FDP haben der jeweilige Vorstand, die Untergliederungen, die Sonderorganisationen und teils die Fachausschüsse Antragsrecht. Dies gilt auch für die Grünen. In beiden Parteien kann auch eine bestimmte Zahl von Delegierten oder Mitgliedern einen Antrag stellen. Während sich bei den Liberalen mit zehn (Land) beziehungsweise 25 (Bund) Delegierten dafür eine größere Gruppe zusammenschließen muss, ist Input der Grünen-Basis quantitativ sehr leicht: Im Land genügt ein einziger Delegierter, in Land und Bund zudem 20 Mitglieder, die nicht einmal Delegierte sein müssen. Auf FDP-Bundesparteitagen müssen sich drei Kreisverbände zusammentun für einen Antrag, bei den Grünen genügt ein einziger Ortsverband.

Ähnliches gilt für das Recht der Vorstände und der Untergliederungen, einen außerordentlichen Parteitag einzuberufen. Auch dieses Kontrollrecht ist bei den Grünen weiter gefasst als bei der FDP: Bei den Liberalen können Basismitglieder einen Parteitag überhaupt nicht einberufen, Untergliederungen nur auf der jeweils höheren Stufe und nur im Zusammenwirken. Bei den Grünen hingegen kann eine relativ geringe Anzahl der Kreisverbände - zehn beziehungsweise 20 Prozent - einen Bundes- beziehungsweise Landesparteitag erzwingen; im Bund auch ein Zehntel der Mitglieder. Auch wenn die Einberufung eines außerordentlichen Parteitages „von unten“ in der Praxis äußerst selten vorkommt, sind die Regelungen doch aufschlussreich. Bei den Grünen dürfen Vorstand und Parteitag einen

30 Vgl. Peter Dausend, FDP-Karrieren - Wenn schon Frauen, dann schöne, in: Die Zeit vom 8. März 2012. 
Sonderparteitag nur mit Zwei-Drittel-Mehrheit einberufen, um Manipulationen „von oben“ zu erschweren. Bei der FDP fällt das zusätzliche Einberufungsrecht der Parlamentsfraktionen auf, was deutlich auf den Ursprung der Liberalen als Honoratiorenpartei verweist.

\subsection{Organisation der Parteitage}

Bei der FDP sind Parteitage oberhalb der Kreisebene Delegiertenversammlungen. In den Landesverbänden der Grünen in Hessen, Berlin, Hamburg und Bremen gibt es hingegen Mitgliederversammlungen, neben oder anstatt Delegiertenversammlungen.

Bei einem Besuch von FDP- und Grünen-Parteitagen ab der Landesebene fällt sofort die unterschiedliche Beratungskultur auf: bei der FDP können sich einzelne Delegierte leicht zu Wort zu melden, denn Dauer und Dichte der Beratungen sind gering. Parteitage enden gegen 19 Uhr, sofern die Debatte nicht per Geschäftsordnungsbeschluss gestrafft wurde. Ganz anders bei den Grünen: Beratungsdauer und -dichte sind hoch, es wird mindestens bis 20 Uhr getagt, auf Bundesdelegiertenkonferenzen mitunter bis weit in die Nacht. Parteitage zur Listenaufstellung haben teils Mühe, die laut Rechtsprechung jedem Kandidaten zustehenden sieben Minuten Vorstellungszeit einzuhalten, weshalb beispielsweise der Dortmunder Parteitag 2009 nur 25 von 99 möglichen Listenplätzen besetzen konnte. ${ }^{31}$ Weil die Zahl der Wortmeldungen regelmäßig die Zahl der angesetzten Redebeiträge übersteigt, wird auf ein Losverfahren zurückgegriffen. Redebeiträge sind bei den Grünen aber „quotiert“, das heißt Männer und Frauen reden abwechselnd. Es gibt daher getrennte Losverfahren. Die Chancen eines einfachen männlichen Grünen-Delegierten, der weder Antragsteller ist, noch als Mandats- oder Funktionsträger einen „gesetzten“ Redebeitrag halten kann, zu sprechen, sind also gering. Dies ist so nicht intendiert, sondern eine Folge des enorm ausgeprägten Redebedürfnisses grüner Delegierter und der stark unterschiedlichen Verteilung dieses Redebedürfnisses über die Geschlechter.

Bei den Grünen haben weibliche Delegierte ein aufschiebendes Vetorecht in „Fragen des Selbstbestimmungsrechts der Frau“ („Frauenvotum“). In der Frühzeit der Grünen konnten Frauen so Beschlüsse zur Frauenpolitik aufhalten, was heute keine Bedeutung mehr hat. Wenn sich für ein Wahlamt aber nicht genügend Kandidatinnen finden, können die Frauen durch ihr Veto bestimmen, ob der Platz für Männer "geöffnet" oder die Wahl zur Suche nach Kandidatinnen auf einen späteren Zeitpunkt verschoben wird. Weitere frühere grüne Spezifika wie das Meinungsbild und die „Paritätische Nicht-Entscheidung“ werden nicht mehr angewandt.

Versammlungen von Delegiertengruppen haben unterschiedlichen Stellenwert: Bei der FDP gibt es solche Delegiertenbesprechungen bei Wahlparteitagen in Land und Bund. Die Bezirks- beziehungsweise Landesverbände treffen sich, um im Vorfeld ausgehandelte Personalpakete zu besprechen. Die Grünen kennen solche Besprechungen nur auf der Bundesdelegiertenkonferenz. Auf der Landesversammlung werden Personalabsprachen nur informell kommuniziert. Eine gewichtige Funktion nehmen bei den Grünen aber die dem eigentlichen Bundesparteitag (und einigen Landesparteitagen) vorgelagerten Strömungstreffen der Linken und der Reformer ein, die Entscheidungen vorstrukturieren. Informelle Zusammen-

31 Vgl. Bündnis 90/Die Grünen, Europaliste 2009, http://www.gruene-partei.de/cms/default/ dok/268/268109.europaliste_2009.htm (Abruf am 28. Juni 2012). 
künfte der Strömungen (Linksliberale, Nationalliberale) gibt es auch am Rande von FDPParteitagen; sie erreichen jedoch nicht ansatzweise die Bedeutung der Grünen-Strömungstreffen.

Die Antragsreihung wurde bei der FDP ursprünglich durch eine Antragskommission wahrgenommen. Die Grünen sortierten Anträge nach Reihenfolge ihres Eingangs. Angesichts der fehlenden demokratischen Legitimation dieser Verfahren gingen Liberale und Grüne vor Kurzem zur Bestimmung durch die Delegierten selbst über. Eine Ausnahme gibt es für Leitanträge des Parteivorstands. Eine grüne Eigenheit hingegen ist das umfangreiche Verhandlungsregime im Vorfeld der Bundesparteitage: In mehreren Aussendungsrunden werden über 1.000 A4-Seiten an alle Delegierten verschickt, da die Untergliederungen und Mitglieder sehr aktiv im Formulieren von Anträgen sind. In mehreren Antragstellertreffen vor und während des Parteitags wird dann versucht, die Zahl der Abstimmungen durch Zusammenführung und Kompromissfindung zu verringern. Die FDP kennt keine Antragstellertreffen, wofür angesichts des viel geringeren Umfangs - ein Antragsbuch ist 250 bis 350 Seiten dick ${ }^{32}$ - aber auch kein Bedarf besteht. Hingegen sind die Parteitage der Grünen durch chronische Überlastung und Zeitmangel geprägt.

\subsection{Alternativen zu ordentlichen Parteitagen}

Der Kleine Parteitag der FDP (Bundes-/Landeshauptausschuss) verlor infolge des Rückgangs programmatisch-ideologischer Auseinandersetzungen an Bedeutung und wurde in den 1990er Jahren abgeschafft. Bei den Grünen gibt es den Kleinen Parteitag (Länderrat) auf Bundesebene noch, vor allem zur programmatischen Diskussion, zur Vernetzung und zur Herstellung von Medienöffentlichkeit vor Landtagswahlen. Weil Delegierte hier Landesund nicht Basisvertreter sind, werden sie auf zwei Jahre gewählt. Auf Landesebene kennen Liberale beziehungsweise Grüne die Kreisvorsitzendenkonferenz beziehungsweise das Kreisvorständetreffen als Koordinierungsgremium. Direktdemokratische Entscheidungsverfahren haben in beiden Parteien unterschiedliche Bedeutung: In der FDP wurde das Instrument trotz Beteiligungsquoren (ein Drittel im Bund, ein Fünftel im Land) immerhin dreimal zur Entscheidung von Sachfragen genutzt. ${ }^{33}$ Bei den Grünen sind Urabstimmungen, zuletzt zur Lockerung der Trennung von Amt und Mandat ${ }^{34}$, selten, obwohl es keine Quoren gibt. Außerordentliche Parteitage als weitere Alternative werden in der FDP in Sachfragen selten, jedoch häufig im Vorfeld von Bundestagswahlen zum Beschluss von Wahlprogramm und Koalitionsaussage genutzt. ${ }^{35}$ Bei den Grünen sind Sonder-Bundesdelegiertenkonferenzen zur Diskussion von Sachfragen hingegen häufig. ${ }^{36}$

32 Vgl. 59. Ordentlicher Bundesparteitag der FDP in München, 31. Mai/1. Juni 2008, Anträge, http://59.parteitag.fdp.de/files/199/Antragsbuch_2008.pdf; 62. Ordentlicher Bundesparteitag der FDP in Rostock, 13. bis 15. Mai 2011, Anträge, http://www.fdp.de/files/1161/Antragsbuch-62. BPT.pdf (Abruf am 28. Juni 2012).

33 Großer Lauschangriff (1995), Wehrpflicht (1997), Euro-Rettungsschirm (2011).

342003 auf Bundes- und 2004/2005 auf Landesebene.

35 Zuletzt zur Aussetzung der Wehrpflicht (2000), im Vorfeld der Bundestagswahlen 2005 und 2009 zur Billigung des Koalitionsvertrags (2009) und zur Euro-Rettung (2011).

36 Zuletzt zur Billigung der Koalitionsverträge (1998/2002), zum Kosovo-Einsatz (1999), zur Agenda 2010 (2003), zu Afghanistan (2007) und zum Atomausstieg (2011). 
Wie die beiden Beispiele Großer Lauschangriff (FDP) und Lockerung der Trennung von Amt und Mandat (Grüne) zeigen, lässt sich die Gesamtheit der Mitglieder leichter von den Argumenten der Parteispitze überzeugen als die auf Parteitagen versammelte „Basis“: Ein auf Parteitagen mehrfach abgelehntes Vorhaben konnte so jeweils plebiszitär durchgesetzt werden. ${ }^{37}$ Dies erklärt sicher auch den unterschiedlichen Gebrauch der beiden Instrumente in den beiden Parteien.

\subsection{Vorstandsformate und -wahlen}

\section{Vorstandsformate ${ }^{38}$}

Die personelle Entscheidungsfunktion gilt als Hauptaufgabe der Parteitage. Beide Parteien wählen die Vorstände auf zwei Jahre, wobei die Formate trotz unterschiedlicher Bezeichnungen und leicht abweichender Zusammensetzung sehr ähnlich sind. Die Vorstände gliedern sich in einen inneren Kreis (FDP: Präsidium, Grüne: Bundes-/Landesvorstand) und einen um weitere Personen (gewählte Beisitzer, Vertreter der Untergliederungen und Vorfeldorganisationen) ergänzten weiteren Kreis (FDP: Bundes-/Landesvorstand, Grüne: Parteirat). Von den 34 Beisitzern des FDP-Bundesvorstands werden 16 auf Vorschlag der Landesverbände („Kurfürsten“) und 18 in freier Wahl („freie Wildbahn“) gewählt. Auch die 13 Beisitzer im Landesvorstand teilen sich auf in sieben auf Vorschlag der Bezirksverbände („Säulenheilige“) und sechs auf der „freien Wildbahn“ gewählte. Auf beiden Ebenen kommen bestimmte Mandatsträger wie Minister, Fraktionsvorsitzende, Vertreter der Vorfeldorganisationen (Frauen, Jugend, Senioren u.a.) und die Vorsitzenden der jeweiligen Untergliederungen ohne Stimmrecht hinzu. Eine solche Kooptierung erfolgt entweder laut Satzung oder auf einen Vorstandsbeschluss hin. Bezirks- und Kreisverbände weisen eine ähnliche Struktur auf, allerdings ohne institutionalisierten Regionalproporz. Bei den Grünen gibt es auf Bundesebene nur eine Teilkongruenz zwischen innerem und äußerem Kreis, weil nur drei der sechs Parteiratsmitglieder automatisch dem Bundesvorstand angehören. Ein Grünen-Spezifikum sind zudem Frauenrat und Finanzrat als eigene Beschlussgremien in frauenpolitischen beziehungsweise finanziellen Angelegenheiten. Auf Kreis- und Bezirksebene finden sich bei den Grünen ganz unterschiedliche, teils deutlich schlankere Strukturen. Zudem existiert kein Bezirksverband Oberbayern. Die Stellung von Generalsekretär beziehungsweise Geschäftsführer unterscheidet sich in beiden Parteien: Der Generalsekretär wird bei der FDP als einziges Vorstandsmitglied auf Vorschlag des Bundes- beziehungsweise Landesvorsitzenden gewählt. Der Politische Geschäftsführer des Grünen-Bundesverbands gehört Parteirat und Vorstand an und wird analog zu den übrigen Mitgliedern vom Bundesparteitag gewählt. Im Gegensatz dazu wird das Amt des Landesgeschäftsführers per Ausschreibung vergeben; er gehört keinem Parteigremium an.

Herrscht bei der FDP eine klare, einfache Struktur vor, gibt es bei den Grünen Dopplungen durch die Doppelspitze und die Trennung von Amt und Mandat: So stand bis 2009

37 Vgl. Jürgen Dittberner, Sind die Parteien noch zu retten?, Berlin 2004, S. 261; Christoph Egle, In der Regierung erstarrt? Die Entwicklung von Bündnis 90/Die Grünen von 2002 bis 2005, in: ders. / Reimut Zohlnhöfer, Ende des rot-grünen Projekts. Eine Bilanz der Regierung Schröder, Wiesbaden 2007, S. 98 - 123, S. $102 \mathrm{ff}$.

38 Vgl. Dietrich Herzog, a.a.O. (Fn. 9). 
mit Guido Westerwelle eine einzige Person an der Spitze von Partei und Fraktion, bei den Grünen hingegen vier Personen (derzeit Claudia Roth, Cem Özdemir, Jürgen Trittin und Renate Künast). Diese Dopplungen haben Vorteile wie die Profilierungsmöglichkeit für Neue und die kontinuierliche Ergänzung des Führungskräftereservoirs, aber auch Nachteile wie Kontinuitätsbrüche, eingeschränkte Personalauswahl und Fehlbesetzungen. ${ }^{39}$ Bei der FDP ist neben Elementen formalisierten Regionalproporzes („Kürfürsten“ und „Säulenheilige“) allenfalls ein gewisser informeller Proporz zugunsten von Frauen, Ostdeutschen und Parteiflügeln festzustellen. Bei den Grünen gibt es in den Vorständen formale Quoten für Frauen und Ostdeutsche. Der wichtige Strömungsproporz im Bund und in vielen Landesverbänden, der Regionalproporz sowie die Berücksichtigung von Migranten sind hingegen informeller Natur.

Auch die Mitarbeit von Mandatsträgern ist in beiden Parteien unterschiedlich ausgeprägt: Bei der FDP erfolgt eine gezielte Einbindung durch Kooptierung; Mehrfachmitgliedschaften in den Vorständen verschiedener Ebenen sind häufig. Die Grünen hingegen begrenzen die Mandatsträgermitwirkung durch die Regelungen zur Vereinbarkeit von Amt und Mandat. Aktuell dürfen nur zwei der sechs Bundesvorstands- und der sechzehn Parteiratsmitglieder Abgeordnete sein, nicht aber Minister oder Fraktionsvorsitzende. In Bayern dürfen zwei der vier Parteirats- und fünf der sechzehn Vorstandsmitglieder ein Mandat innehaben. In den übrigen Landesverbänden gibt es ein breites Spektrum, das von der unverändert strikten Trennung von Amt und Mandat (Berlin) bis hin zur vollen Vereinbarkeit (Hessen) reicht. (Baden-Württembergs Grüne beschlossen nach der Übernahme des Ministerpräsidentenamtes 2011 eine Sonderregelung, so dass der grüne Regierungschef beratend an den Vorstandssitzungen teilnimmt.) In der Satzung des Landesverbands Bayern gibt es zudem ein Inkompatibilitätsgebot: Personen dürfen den Vorständen auf höchstens zwei Ebenen angehören. Bei den Grünen treten mehr oder weniger stark institutionalisierte Koordinierungstreffen, zum Beispiel zwischen Landesvorstand und Landtagsfraktion, an die Stelle der bei der FDP üblichen Kooptierung. Dort sind zudem Parteiämter bis auf die wichtige Ausnahme des Generalsekretärs auf Bundesebene Ehrenämter. Bei den Grünen hingegen werden die Mitglieder des kleineren Vorstandskreises, also von Bundes- und Landesvorstand, für ihre Arbeit im Unfang von bis zu einem Akademiker-Vollzeitgehalt bezahlt. Dieser Kontrast zwischen formaler Mandatsträgermitwirkung und Ehrenamt einerseits und Mandatsträgerausschluss und Hauptamt andererseits verweist wiederum auf die unterschiedlichen Wurzeln der beiden Parteien als Honoratiorenpartei beziehungsweise außerparlamentarische Bewegung.

FDP und Grüne erneuern ihre Bundesvorstände alle zwei Jahre komplett. Bei Nachwahlen dauert die Amtszeit bis zur nächsten regulären Neuwahl. Folglich teilen sich die Parteitage in Wahlparteitage und Arbeits- oder programmatische Parteitage auf. Auf Landesebene sind Vorstandswahlen der Grünen hingegen Teilerneuerungswahlen. Dieser Modus entstand durch zufällig notwendig gewordene Nachwahlen, wurde aber bewusst beibehalten, um die Kontinuität zu sichern. Parteitage sind folglich immer Mischtypen, da jedes Jahr sowohl Wahlen als auch programmatische Diskussion anstehen.

39 Vgl. Thomas Poguntke, Die Bündnisgrünen in der babylonischen Gefangenschaft der SPD, in: Oskar Niedermayer (Hrsg.), Die Parteien nach der Bundestagswahl 1998, Opladen 1999, S. 83 101. 


\section{Vorstandswahlen}

Die FDP setzt keine automatisierten Wahlverfahren ein. Die bayrische Landesgeschäftsstelle befürwortet elektronische Abstimmungen aus Praktikabilitätsgründen, die Landesvorsitzende Sabine Leutheusser-Schnarrenberger lehnt diese aus politischen Erwägungen hingegen ab. Interessant ist, dass die Grünen, die der elektronischen Datenverarbeitung seitens des Staates ebenso kritisch gegenüberstehen, seit 2005 bei parteiinternen Wahlen auf Bundesebene und zur Aufstellung der Europawahlliste auf elektronische Wahlverfahren zurückgreifen. Im Landesverband Bayern kommen diese infolge eines Parteitagsbeschlusses seit 2009 aber nur noch bei der Aufstellung der Bundestagsliste zum Einsatz.

Durch das Zusammenspiel von Frauenquote, Doppelspitze und Trennung von Amt und Mandat sind Grünen-Parteitage bei der Personalauswahl sehr eingeschränkt. Im Konfliktfall ist eine weibliche Abgeordnete im Vorteil: Da die Frauenplätze immer als erste besetzt werden, hat ein männlicher Abgeordneter das Nachsehen, wenn das Mandatsträgerkontingent bereits ausgeschöpft ist. Die Wahlreihenfolge determiniert Ämter also „zufällig“, ohne dass eine Ämterkumulation bei Frauen herrschaftstheoretisch als weniger problematisch als bei Männern konstatiert worden wäre. Bei der FDP, die weder Frauenquote noch Doppelspitze oder die Trennung von Amt und Mandat kennt, ist die Kandidatenauswahl allenfalls durch informelle Quotenerwägungen eingeschränkt. Während bei den Freidemokraten die Wiederwahl von Amtsträgern die Regel ist, ist bei den Grünen die Nicht-Wiederwahl zumindest eine realistische Option, wie das Scheitern Fritz Kuhns bei der Wahl in den Parteitrat 2008 oder die Nicht-Aufstellung der früheren Parteichefin Angelika Beer 2009 zeigen.

Eine grüne Besonderheit ist der tradionelle Rückgriff auf ausführliche, an alle Delegierten in Papierform ausgegebene, mit Foto und Lebenslauf versehene Bewerbungen im Vorfeld von Wahlen. Die FDP macht hiervon erst in letzter Zeit und nicht durchgängig Gebrauch. Früher waren bei den Grünen Kandidaten zudem ohne „Basisvotum“ des jeweiligen Kreisoder Landesverbands chancenlos, heute spielt dies eine geringere Rolle.

\section{Vergleichende Analyse}

Die Unterschiede der Entscheidungs- und Repräsentationskultur beider Parteien sind vor allem auf der mittleren (propagierte Werte) und unteren (unausgesprochene Grundannahmen) Ebene der Organisationskultur angesiedelt. Auf der unteren Ebene sind sie, eben weil sie unausgesprochen sind, schwieriger zu fassen und zu interpretieren.

\subsection{Gemeinsamkeiten}

Vor allem bei den Rahmenbedingungen finden sich Gemeinsamkeiten: Die Parteiorganisation (Mitgliederzahl, Organisationsgrad), das Vorstandsformat und die Organisation der Parteitage (Dauer, Häufigkeit, Delegiertenzahl, Wochentage, Ablauf, Umfeld, Problemlagen, Kriterien zur Auswahl des Tagungsortes) sind ähnlich. Aber auch die Spezifika der Entscheidungs- und Repräsentationskultur weisen Gemeinsamkeiten aus. So spielen direktdemokratische Verfahren zur Entscheidung von Sachfragen in beiden Parteien keine größere Rolle. Sie stellen jedoch ein probates Mittel der Spitze dar, um in Ausnahmesituationen Parteitagsentscheidungen mithilfe der Mitgliedschaft revidieren zu können. Auffallend ist zudem, dass 
beide Parteien in den letzten Jahren dazu übergegangen sind, die Antragsreihenfolge von den Delegierten selbst festlegen zu lassen. FDP und Grüne ähneln sich außerdem in einer Reihe kleinerer Details, in denen sie sich wiederum von den Volksparteien CDU/CSU und SPD unterscheiden: Dies reicht von der Abhaltung von Parteitagen an Wochenenden (Union und SPD versammeln sich angesichts der großen Zahl Hauptamtlicher unter den Delegierten an Wochentagen) bis hin zur Bestimmung aller aussichtsreichen Listenplätze im Vorfeld öffentlicher Wahlen in einzelnen Wahlgängen (bei den Großparteien werden oft Listenvorschläge en bloc abgestimmt).

\subsection{Unterschiede}

Während die Regelungen zur Entscheidungsfindung bei der FDP hochgradig kohärent sind, finden sich bei den Grünen oft historisch entstandene, aber bewusst beibehaltene, abweichende Regelungen zwischen Bundes- und Landesverband und zwischen verschiedenen Landesverbänden. Wahl- und Abstimmungsergebnisse sind bei der FDP in der Regel vorhersehbar, ganz im Sinne der oligarchischen Interpretation innerparteilicher Demokratie, wonach die erneute Delegierung von Macht als ein Recht der Amtsinhaber wahrgenommen wird. ${ }^{40}$ Wenn prominente Kandidaten einmal im ersten Wahlgang die Mehrheit verfehlen, werden sie nach eindringlichen Appellen beim zweiten Versuch doch gewählt. ${ }^{41}$ Ein sachpolitisches Veto, wie etwa die Ablehnung des Großen Lauschangriffs ist die Ausnahme. Bei den Grünen sind die Ergebnisse von Wahlen und Abstimmungen teils offen, wie Personalentscheidungen, aber auch der anhaltende Widerstand gegen die Aufhebung der Trennung von Amt und Mandat zeigen. Die FDP nutzt, wenn überhaupt, den tendenziell die Parteispitze begünstigenden Mitgliederentscheid als alternatives Entscheidungsverfahren zu ordentlichen Parteitagen, die Grünen hingegen Sonderparteitage, die mittlere Funktionäre begünstigen.

Die Liberalen können es sich angesichts überschaubaren Diskussionsbedarfs leisten, auf elektronische Wahlverfahren zu verzichten. Bei den Grünen werden sie infolge hoher Beratungsdichte trotz inhaltlicher Bedenken eingesetzt. Die FDP benötigt zudem kein ausgeprägtes Verhandlungsregime im Vorfeld von Parteitagen, wie es bei den Grünen angesichts der schieren Masse notwendig ist. Den Strömungskonsens als Grundlage wichtiger Entscheidungen auf Bundesebene gibt es nur bei den Grünen, da die Parteiflügel der FDP nicht die Bedeutung der Grünen-Strömungen erreichen. Nur die Grünen führen getrennte Rednerlisten für Männer und Frauen. Auf FDP-Parteitagen haben nur Delegierte Rederecht, können dieses faktisch aber auch wahrnehmen, während bei den Grünen alle Mitglieder ein Rederecht haben, das in der Praxis aber aufgrund des Losverfahrens nur schwer einzulösen ist. Das Antrags- und Einberufungsrecht ist in der FDP eher eng, bei den Grünen eher weit gefasst. Die unterschiedlich ausgeprägten Rechte von Parlamentsfraktionen beziehungsweise Parteibasis verweisen auch hier auf den unterschiedlichen Ursprung als Honoratiorenbeziehungsweise Anti-Parteien-Partei.

40 Vgl. Robert Michels, a.a.O. (Fn. 3).

41 Vgl. „Kubicki im zweiten Durchgang in FDP-Vorstand gewählt“, in: Welt.de vom 7. Mai 2005, http://www.welt.de/print-welt/article669458/Kubicki-im-zweiten-Durchgang-in-den-FDP-Vorstand-gewaehlt.html (Abruf am 28. Juni 2012). 
Die FDP-Entscheidungsstrukturen zeichnen sich also durch Klarheit und Kohärenz aus. Mit Ausnahme der Zweidrittelmehrheit für Satzungsänderungen gilt das einfache demokratische Mehrheitsprinzip. Grüne Entscheidungsstrukturen sind hingegen komplex und heterogen. Das demokratische Mehrheitsprinzip wird mehrfach relativiert oder, je nach Sichtweise, ergänzt, und zwar durch den im Bund faktisch notwendigen Strömungskonsens, den theoretisch vorgesehenen Geschlechterkonsens und das quasiföderalistische Prinzip, wie es in der Überrepräsentation kleiner Kreisverbände und dem daraus folgenden ungleichen Stimmengewicht der einzelnen Parteimitglieder zum Ausdruck kommt.

FDP-Bundesparteitage lassen sich kaum als Basisvertetung im engeren Sinne charakterisieren: Nur ein Teil der Kreisverbände entsendet Delegierte. Wahlkriterium für das Delegiertenamt ist die Bekanntheit auf Bezirksebene. In Bayern vertritt ein Bundesparteitagsdelegierter 62 Mitglieder und mehr. Bei den Grünen hingegen sind auch Bundesdelegiertenkonferenzen echte Basisvertretungen. Alle Kreisverbände verfügen über ein Grundmandat. Wahlkriterium ist die Verwurzelung vor Ort. Im kleinsten bayerischen Kreisverband repräsentiert ein Delegierter nur 18 Mitglieder.

Bei der FDP ist das Delegiertenamt ein persönliches Amt, das auf Zeit vergeben wird, ungeteilt ausgeübt wird und übertragbar ist. Eine Rechenschaftspflicht gibt es nicht. Kosten werden nur teilweise und indirekt erstattet. Bei den Grünen hingegen ist das Delegiertenamt ein funktionales Mandat. Das kommt bereits bei der nur für eine Versammlung erfolgenden Wahl zum Ausdruck. Die Delegiertenstimme ist nicht übertragbar. Grünen-Delegierte müssen im Kreisverband unter Umständen Rechenschaft ablegen. Die Kostenerstattung ist hingegen direkt und umfassend. Gemäß der Bedeutung der Strömungen bei den Grünen wird das Parteitagsplenum hier durch Delegiertenbesprechungen der regionalen Gliederungen und der Strömungen ergänzt, das der FDP nur durch Delegiertenbesprechungen der regionalen Untergliederungen. FDP-Vorstände sind groß. Mandatsträger, aber auch regionale und funktionale Untergliederungen der Partei, sind durch das System der Kooptation breit eingebunden. Grüne Vorstände sind hingegen strukturell schlank. Durch die nur teilweise gelockerte Trennung von Amt und Mandat sind Mandatsträger nur durch informelle Koordinationstreffen eingebunden. Regionalproporz ist bei der FDP durchgängig formalisiert, bei den Grünen hingegen überwiegend informell. Sowohl vertikal (Bund - Land usw.) als auch horizontal (zum Beispiel Partei - Fraktion) zeichnen sich die Liberalen eher durch Posititionsverflechtung (Ämterkumulation), die Grünen eher durch Positionstrennung aus. ${ }^{42}$

Parteiämter sind bei der FDP anders als bei den Grünen Ehrenämter. Faktisch hat aber ein großer Teil der Funktionsträger öffentlich besoldete Mandate inne. Dem völligen Verzicht auf Quoten bei der FDP steht eine Vielzahl von Quotierungen (Frauenquote, Ostdeutschenquote, informelle Migrantenquote) bei den Grünen gegenüber. Parteitage der Freidemokraten sind durchgehend Delegiertenversammlungen, bei den Grünen mitunter Mitgliederversammlungen. Anders als die FDP haben die Grünen den Kleinen Parteitag nicht abgeschafft; das Gremium ist jedoch von untergeordneter Bedeutung. Die FDP hält nur Gesamterneuerungswahlen ab, die Grünen je nach Ebene Gesamt- oder Teilerneuerungswahlen.

Die Repräsentationskultur der FDP ist also, wie in der Hypothese formuliert, vom Modell der klassischen Repräsentativdemokratie geprägt. Weniger die Basis denn die Funktio- 
näre mittlerer Ebene stellen die Delegierten. Der Ursprung der FDP als Honoratiorenpartei ist darin ebenso erkennbar wie das klassische liberale Repräsentationsverständnis, etwa die Vorstellung vom freien Mandat. Bei den Grünen ist das ursprüngliche Modell der Basisdemokratie, auch wenn es sich stark der Repräsentativdemokratie angenähert hat, noch in vielen Elementen nachweisbar. Delegierte sind hier viel stärker echte Basisvertreter. Der Ursprung der Grünen als außerparlamentarische Bewegung und das frühere Selbstverständnis als Anti-Parteien-Partei sind, ebenfalls entsprechend der anfänglichen Hypothese, noch sichtbar. Das alternative, sich dem imperativen Mandat annähernde Modell der Basisdemokratie ist hier zumindest teilweise noch wirkmächtig.

Für die FDP werden wichtige Annahmen der Oligarchisierungsthese bestätigt. Bei den Grünen lässt sich eine Oligarchisierungstendenz dann feststellen, wenn die Ansprüche aus der Frühzeit der Grünen den Maßstab bilden. Allerdings ist es bemerkenswert, in welchem Maße machtbeschränkende und machtkontrollierende Elemente trotz des Annäherungsund Normalisierungsprozesses der Grünen erhalten geblieben sind. Aber auch Joachim Raschkes Feststellung gilt angesichts der nach wie vor beibehaltenen Trennung von Amt und Mandat, dass der Hauptunterschied zwischen den Grünen und den übrigen Parteien darin bestehe, dass Parteitagsdemokratie und anderswo, vor allem in der Bundestagsfraktion und auf den Strömungstreffen vorgenommene Weichenstellungen auseinanderfallen. ${ }^{43}$

\section{Wirkungen von Entstehungshintergrund und Selbstverständnis auf Repräsentations- und Entscheidungskulturen von Parteien}

Für FDP wie Grüne lässt sich feststellen, dass der unterschiedliche Entstehungshintergrund und das unterschiedliche Selbstverständnis in einer unterschiedlichen Repräsentations- und Entscheidungskultur resultieren. Die FDP ist durch ihren Ursprung als Honoratiorenpartei geprägt und ist von Ideen der klassischen Repräsentativdemokratie geleitet. Bei den Grünen ist ihr Ursprung als Anti-Parteien-Partei und parlamentarischer Arm der Neuen Sozialen Bewegungen sichtbar; sie orientieren sich noch immer an einem, wenn auch modifizierten Basisdemokratie-Modell. Neben Unterschieden auf der oberen Kulturebene des sichtbaren Verhaltens, etwa in der Dichte der Antragsberatung, wurden auch Verschiedenheiten auf der mittleren und unteren Organisationskultur-Ebene der propagierten Werte und der unausgesprochenen Grundannahmen deutlich. Die Grenzziehung zwischen beiden ist mitunter schwierig: So können das einfache Demokratieprinzip und der Verzicht auf Frauenquoten bei der FDP beziehungsweise die Relativierung des Demokratieprinzips zum Zwecke einer besseren Basis- und Frauenvertretung und die Quotierung der Redebeiträge bei den Grünen als propagierte Werte wie auch als unausgesprochene Grundannahme gesehen werden, je nachdem, wie oft die entsprechenden Prämissen bewusst reflektiert werden. Das ist bei den Frauenrechten der Grünen, die parteiintern mitunter kritisiert werden, sicher häufiger der Fall als bei der Organisation der FDP. In jedem Fall spiegeln die Entscheidungs- und Repräsentationskulturen, dokumentiert durch die Parteitage, die unterschiedliche Herkunft und das ungleiche Selbstverständnis der Parteien wider. 
FDP und Grüne sind ein gutes Beispiel dafür, welche Variationen hinsichtlich der Repräsentation und der Entscheidungsfindung auf Parteitagen trotz der vereinheitlichenden Wirkung von Gesetzgebung, Rechtsprechung und den Erfordernissen der modernen (Medien-) Demokratie möglich sind. Dies betrifft zum einen Unterschiede innerhalb einer Partei, wie die Grünen zeigen, aber auch zwischen den Parteien, wo wiederum die durch Geschichte, unterschiedlichen Status und Größe bedingten Diskrepanzen zwischen den kleinen beziehungsweise mittleren Parteien einerseits und den großen Volksparteien andererseits sichtbar werden.

Diese Ergebnisse sind ein guter Ausgangspunkt für weitere Forschungen: So ließe sich genauer untersuchen, inwieweit die organisatorisch-kulturellen Unterschiede auf unterschiedliche ideologische Standpunkte, unterschiedlichen Entstehungshintergrund und -zeitpunkt oder eine Kombination hiervon zurückzuführen sind. Auch eine genauere Analyse von wichtigen Aspekten der Entscheidungskultur wie Verhandlungen und informellen Gesprächen wäre aufschlussreich. Zudem bleibt die weitere Entwicklung der Piratenpartei abzuwarten. Im Falle einer dauerhaften Etablierung wird es hochinteressant sein, zu sehen, wie und in welchem Umfang dieser neue Akteur seinen innovativen organisatorischen Ansatz, dessen Forderung nach Transparenz frappierend an die frühen Grünen erinnert, beibehalten (können) wird. Die ersten parteiinternen Diskussionen rund um das Mandatsverständnis und die Bezahlung von Vorständen lassen auf einen den Grünen nicht unähnlichen Anpassungsdruck schließen.

\title{
Der Mundatwald bleibt deutsch! Wie das deutsch-französische Grenzabkommen vom 31. Juli 1962 am Auswärtigen Ausschuss des Bundestages scheiterte*
}

\author{
Volker Pilz
}

Der bislang einzige Fall, in dem die Zustimmung zu einem von der Bundesregierung ausgehandelten völkerrechtlichen Vertrag vom Bundestag verweigert wurde, ist das deutschfranzösische Grenzabkommen vom 31. Juli 1962. Diesem Abkommen zufolge sollte ein etwa sieben Quadratkilometer großes Gebiet in der Südpfalz, der Mundatwald, an Frankreich abgetreten werden. Der Auswärtige Ausschuss des Bundestages verhinderte die Ratifizierung des Vertrages, indem er es nach umfangreichen Beratungen unterließ, das Zustimmungsgesetz dem Plenum zur endgültigen Beschlussfassung vorzulegen. Dieser bislang einzige Fall verweigerter parlamentarischer Zustimmung bei der Ratifizierung völkerrechtlicher Verträge gemäß Art. 59 Abs. 2 Satz 1 GG soll im Folgenden näher beleuchtet werden.

* Der Autor dankt Edouard Michel, Ständiger Vertreter der französischen Nationalversammlung bei der Europäischen Union, für seine Unterstützung bei der Dokumentenrecherche. 